\title{
ON THE SUPERPOSITION OF A HEAT SOURCE AND CONTACT RESISTANCE*
}

\section{By S. A. SCHAAF (New York University)}

1. Introduction. The usual condition for a thermal contact resistance is that the temperature discontinuity $T_{1}-T_{2}$ across the interface of two heat conductors [1] and [2] should be proportional to the rate of heat transfer $H$ per unit area there, i.e.

$$
T_{1}-T_{2}=R H, \quad R=\text { "resistance" constant. }
$$

If heat is generated at the same interface, however, the quantity $H$ in (1) cannot be interpreted unless a more specific physical model of the interface is considered. For a

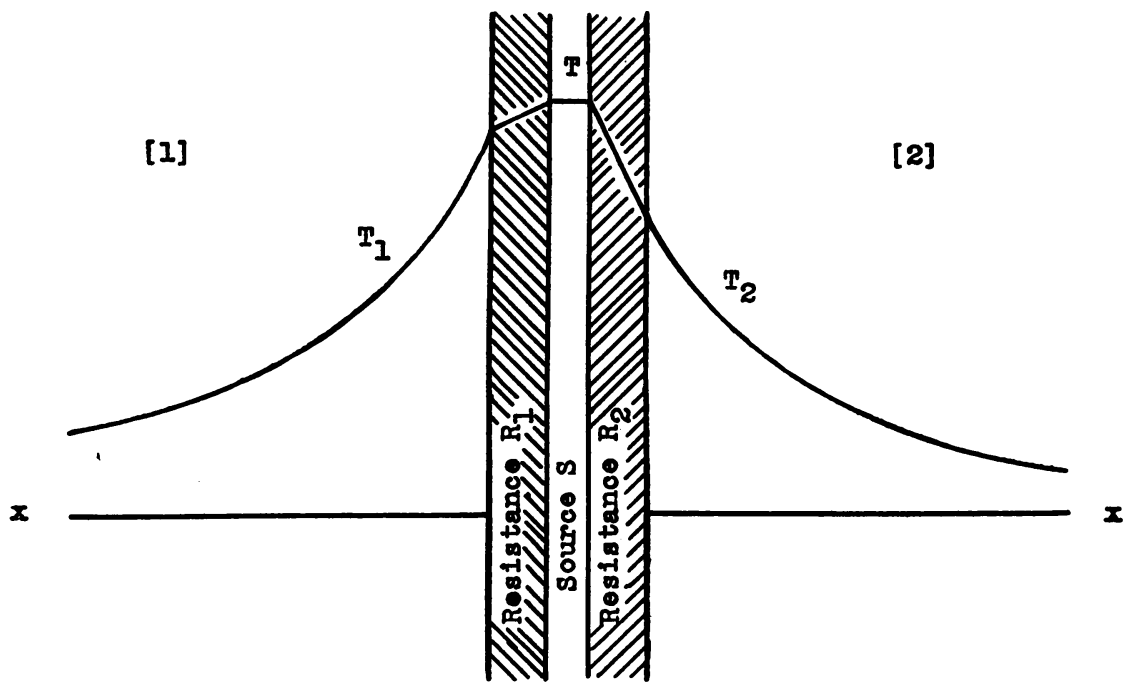

FIG. 1. Magnified view of "lubricated friction" model for interface, consisting in a source $S$ between the two media and separated from them by contact resistances $R_{1}$ and $R_{2}$. A typical temperature distribution for some fixed time $t>0$ is superimposed.

large class of problems, including those in which the heat source is caused by friction, appropriate physical models of the interface lead to a condition of the form

$$
T_{1}-T_{2}=R\left(C_{1} S-H_{1}\right)=-R\left(C_{2} S-H_{2}\right) \quad C_{1}+C_{2}=1,
$$

where $S$ is the rate of heat generated per unit area at the interface, $H_{i}$ is the rate of heat per unit area flowing into the corresponding medium and $C_{i}$ is a constant whose interpretation depends on the nature of the interface. Condition (2) is obtained by considering models of the interface in which either the contact resistance or the heat source is broken up into two parts. If the source is caused by friction as the two solids slide against each other with the interface as slip plane, these two models correspond respectively to lubricated and to dry friction.

* Received May 27, 1946.

1 W. A. Mersman, Heat conduction in an infinite composite solid with an interface resistance, Trans. Amer. Math. Soc. 53, 14-24 (1943). 
Lubricated friction. Here we suppose (see Fig. 1) that the heat source is located between two known contact resistances $R_{1}$ and $R_{2}$, with $R_{1}+R_{2}=R$. This is the case if the two solids are separated by a layer of lubricating fluid, for example melted material from one of the solids. Heat is then generated in the turbulent fluid and flows to the two solids through film contact resistances of the ordinary kind. If we suppose that the fluid temperature is $T$, then according to (1) we have

$$
T-T_{i}=R_{i} H_{i} \quad \text { at } \quad x=0, \quad(i=1,2) .
$$

Eliminating $T$, using $R_{1}+R_{2}=R$ and $H_{1}+H_{2}=S$, we obtain

$$
T_{1}-T_{2}=R_{2} S-R H_{1}=-\left(R_{1} S-R H_{2}\right) \quad \text { at } \quad x=0,
$$

which is equivalent to (2) if we put $C_{i}=1-R_{\mathfrak{\imath}} / R$.

Dry friction. An alternative model for the interface (see Fig. 2) is to suppose that

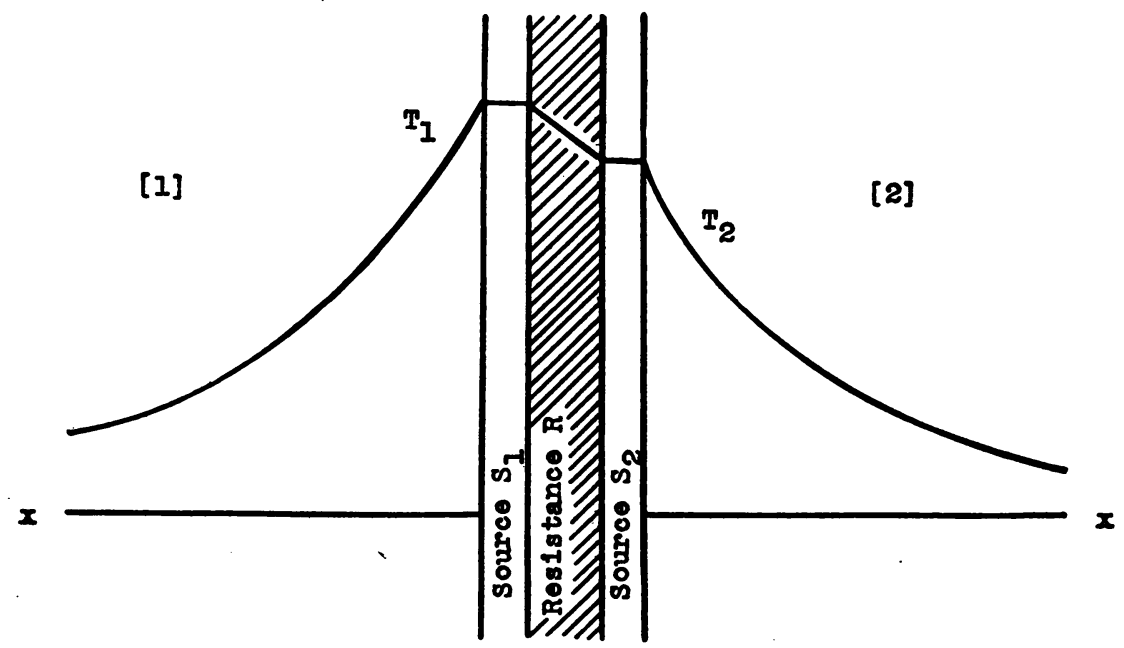

Fig. 2. Magnified view of "dry friction" model for interface, consisting in two sources $S_{1}$ and $S_{2}$ separated by a contact resistance $R$, with typical temperature distribution as some fixed time $t>0$.

the heat is generated on each of the two surfaces, so that there are two sources $S_{1}(t)$ and $S_{2}(t)$, with $S_{1}+S_{2}=S$, separated by a single contact resistance $R$. This seems a reasonable model for dry friction, where the heat is generated on the two sliding surfaces which are separated at most points by a very thin air gap which constitutes the contact resistance. It further seems reasonable to suppose that these two sources are proportional, so that

$$
S_{1}(t)=C_{1} S(t), \quad S_{2}(t)=C_{2} S(t), \quad C_{1}+C_{2}=1,
$$

where $C_{i}$ is an empirical constant depending on the elastic and cohesive properties of the two media and on the geometry of the surface roughness, but not on the source strength $S(t)$ nor the temperature. The rate of heat per unit area flowing across the resistance from [1] to [2] is then $S_{1}(t)-H_{1}(t)$, so that according to (1) we have

$$
T_{1}-T_{2}=R\left(S_{1}-H_{1}\right)=R\left(H_{2}-S_{2}\right) \text { at } x=0 \text { since } S_{1}+S_{2}=H_{1}+H_{2} \text {. }
$$

This is equivalent to (2) providing that the assumption $S_{i}=C_{i} S$ is valid. 
In Sec. 2 we solve a typical heat transfer problem whose formulation involves the boundary condition (2).

2. Problem and solution. Let us consider the case of two semi-infinite media [1] and [2], initially at zero temperature and in contact along the plane $x=0$, where there is a heat source $S(t)$ and a contact resistance $R$. Then, in the usual notation, we have the following boundary value problem:

$$
\begin{gathered}
\frac{\partial^{2} T_{i}}{\partial x^{2}}=\frac{1}{\alpha_{i}} \frac{\partial T_{i}}{\partial t}, \quad(i=1,2), \quad x>0, \quad t>0 . \\
T_{i}=0, \quad t=0, \quad x>0 . \\
T_{1}-T_{2}=R\left[C_{1} S(t)+K_{1} \frac{\partial T_{1}}{\partial x}\right]=-R\left[C_{2} S(t)+K_{2} \frac{\partial T_{2}}{\partial x}\right], \\
x=0, \quad t>0, \quad C_{1}+C_{2}=1
\end{gathered}
$$

Equation (5) is the same as (2) since

$$
H_{i}=-K_{i} \frac{\partial T_{i}}{\partial x} \quad \text { at } \quad x=0, \quad t>0 .
$$

The solution of (3) and (4) in terms of the undetermined functions $H_{i}(t)$ is well known ${ }^{2}$ to be

$$
T_{i}=\beta_{i} \int_{0}^{t} H_{i}(t-u) \exp \left(-y_{i}^{2} / 4 u\right) d u / \sqrt{u},
$$

where $y_{i}=x_{i} / \sqrt{\alpha_{i}}, \beta_{i}=\sqrt{\alpha_{i}} / K_{i} \sqrt{\pi}$. The functions $H_{i}(t)$ may then be determined by use of the Laplace transform from the integral equation (5) and the additional requirement

$$
H_{1}(t)+H_{2}(t)=S(t),
$$

which is obtained by equating the two right members in (5) and then using (6). We denote by $f^{*}(s)$ the transform of $f(t)$, i.e.

$$
f^{*}(s)=\int_{0}^{\infty} e^{-s t} f(t) d t
$$

and recall that

$$
\left[\int_{0}^{t} f(t-u) g(u) d u\right]^{*}=f^{*}(s) \cdot g^{*}(s) .
$$

Transforming (7), we obtain

since $^{3}$

$$
T_{i}^{*}=\beta_{i} H_{i}^{*}(s) \cdot \sqrt{\pi / s} \exp \left(-y_{i} \sqrt{s}\right),
$$

$$
\left[\frac{\exp \left(-y_{i}^{2} / 4 t\right)}{\sqrt{t}}\right]^{*}=\sqrt{\pi / s} \exp \left(-y_{i} \sqrt{s}\right) .
$$

${ }^{2}$ H. S. Carslaw, Conduction of heat in solids, p. 153.

${ }^{3}$ J. Cossar and A. Erdelyi, Dictionary of Laplace transforms, ACS-DSRE No. 71, p. vi-20. 
The transforms of equations (5) and (8) are

$$
\begin{gathered}
\beta_{1} H_{1}^{*} \sqrt{\pi / s}-\beta_{2} H_{2}^{*} \sqrt{\pi / s}=R\left(C_{1} S^{*}-H_{1}^{*}\right), \\
H_{1}^{*}+H_{2}^{*}=S^{*} .
\end{gathered}
$$

Solving for $H_{1}^{*}$, we obtain

$$
H_{1}^{*}=\frac{\beta_{2} \sqrt{\pi}+R C_{1} \sqrt{s}}{\left(\beta_{1}+\beta_{2}\right) \sqrt{\pi}+R \sqrt{s}} S^{*}:
$$

We will treat the cases $S=$ constant $S_{0}$, and $S=S(t)$ separately. In the former case, we have

$$
S^{*}=S_{0} / s,
$$

and the inverse of (12) is then known $n^{4}$ to be

$$
H_{1}(t)=\frac{\beta_{2} S_{0}}{\beta_{1}+\beta_{2}}+\frac{S_{0}\left(\beta_{1} C_{1}-\beta_{2} C_{2}\right)}{\beta_{1}+\beta_{2}} \cdot \exp \left[\left(\beta_{1}+\beta_{2}\right)^{2} \pi t / R^{2}\right] \operatorname{erfc}\left[\left(\beta_{1}+\beta_{2}\right) \sqrt{\pi t} / R\right] \text {, }
$$

where

$$
\operatorname{erfc}(x)=\frac{2}{\sqrt{\pi}} \int_{x}^{\infty} e^{-u^{2}} d u .
$$

$H_{2}(t)$ may be obtained from (14) by interchanging subscripts 1 and 2; the expressions for $T_{i}$ then follow immediately from (7). We observe that as $t \rightarrow \infty, H_{i}$ approaches the constant value it would have for $R=0$, i.e. for no contact resistance. It follows that, although

$$
T_{i}(0, t) \sim \text { const. } \sqrt{t} \text { as } t \rightarrow \infty,
$$

the temperature discontinuity

$$
T_{1}(0, t)-T_{2}(0, t) \rightarrow \text { const. as } t \rightarrow \infty .
$$

For the case $S=S(t)$, we substitute (12) into (7)* to obtain

$$
T_{1}^{*}=\beta_{1} S^{*}\left[\frac{\beta_{2} \sqrt{\pi}+C_{1} R \sqrt{s}}{\left(\beta_{1}+\beta_{2}\right) \sqrt{\pi}+R \sqrt{s}} \cdot \frac{\sqrt{\pi} \exp \left(-y_{1} \sqrt{s}\right)}{\sqrt{s}}\right] .
$$

This is in the form of a product of two functions with known inverses, ${ }^{5}$ so that (10) may be used to obtain

$$
\begin{aligned}
T_{1}= & \beta_{1} \int_{0}^{t} S(t-u) \cdot\left\{\frac{C_{1} \exp \left(-y_{1}^{2} / 4 u\right)}{\sqrt{u}}+\frac{\pi\left(\beta_{2} C_{2}-\beta_{1} C_{1}\right)}{R}\right. \\
& \left.\cdot \exp \left[\frac{\beta_{1}+\beta_{2}}{R} y_{1} \sqrt{\pi}+\frac{\left(\beta_{1}+\beta_{2}\right)^{2}}{R^{2}} \pi u\right] \cdot \operatorname{erfc}\left[\frac{\beta_{1}+\beta_{2}}{R} \sqrt{\pi u}+\frac{y_{1}}{2 \sqrt{u}}\right]\right\} d u .
\end{aligned}
$$

The expression for $T_{2}$ may be obtained from (16) by interchanging the subscripts 1

4. Cossar and A. Erdelyi, loc. cit., p. vi-76.

5 J. Cossar and A. Erdelyi, loc. cit., pp. vi-77, 78. 


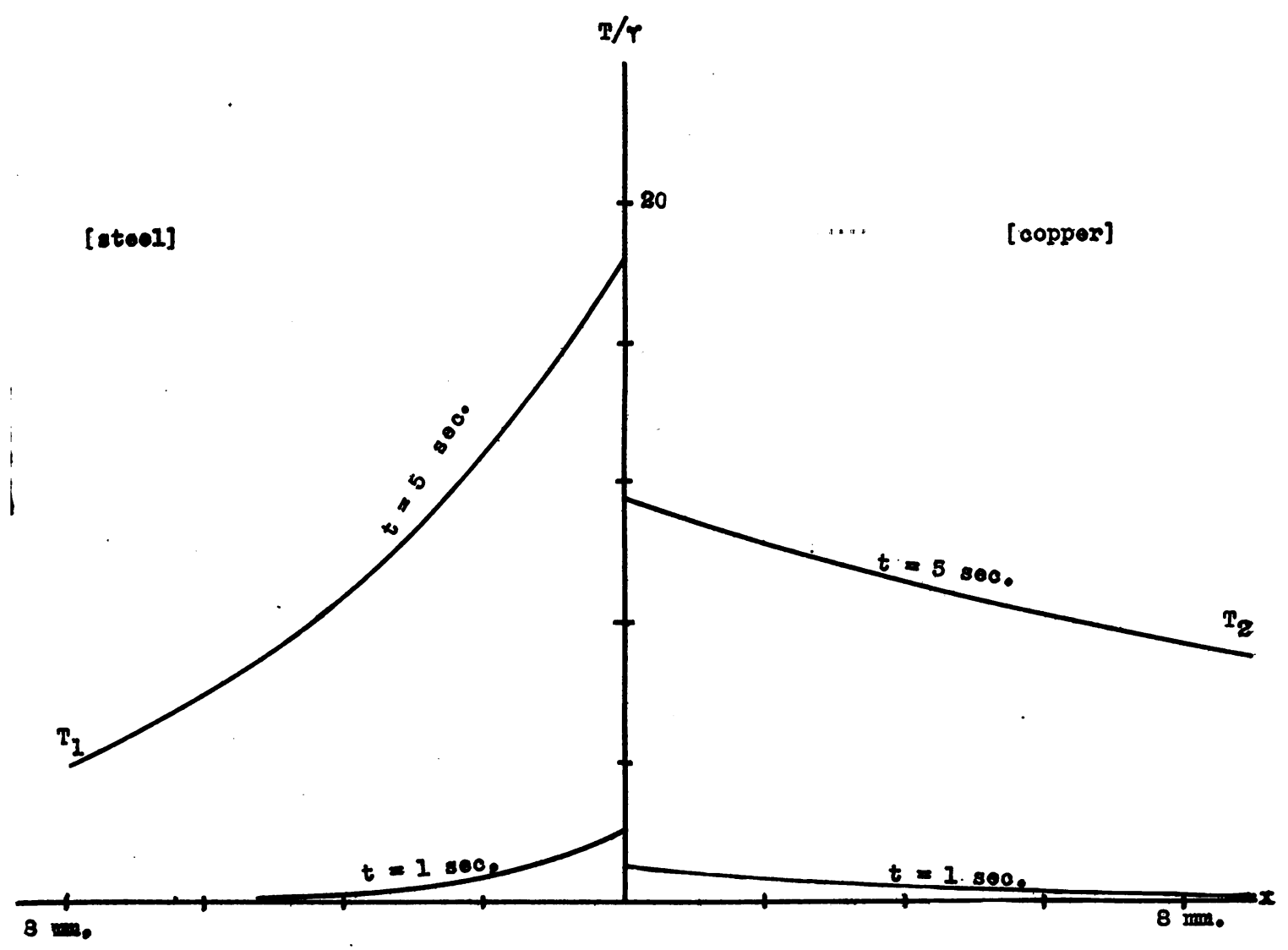

FIG. 3. Temperatures distribution in steel and copper media, separated by a contact resistance and a heat source whose strength is proportional to the time, $S=\gamma t$.

and 2. The evaluation of (16) is given graphically in Fig. 3 for the typical numerical case (in c.g.s.) $\beta_{1}=1.8$ (steel), $\beta_{2}=.6$ (copper), $C_{1}=C_{2}=1 / 2, R=4.2, S(t) \sim t$; which corresponds approximately to the early stages of heat transfer between a steel gun barrel and a copper shell under constant acceleration. 